\title{
The eye and the brain in non-accidental injury involving young children
}

Intracranial injuries in young children, especially those less than 3 years old, which have no obvious explanation and frequently have no external evidence of trauma to the skull, should raise the suspicion of whiplash injury. Whiplash injury is caused by forcible shaking of a young child usually by a member of the family or occasionally. by a childminder. The association between this type of injury and subdural haematoma was first noted by the American radiologist, Professor J Caffey. ${ }^{1}$ There has been much argument since as to whether the various intracranial haemorrhages encountered in non-accidental injury (NAI) in young children are due to shaking or to impact injuries. $^{2} 3$ The generally held view seems to be that either or both mechanisms may be responsible in an individual case. All the 13 cases described by Hadley et $a l^{2}$ were very young (median age 3 months) but none showed any evidence of skull fracture and the authors concluded that these injuries were the result of shaking. Duhaime et $\mathrm{al}^{3}$ found skull fractures in all 13 of the fatalities in their series of 48 infants and young children.

The matter of cause of non-accidental brain haemorrhage has long bedevilled the expert witness in criminal proceedings involving NAI. A question which is frequently put to the medical expert is that concerning the amount of force which would be required to cause intracranial haemorrhages. To some extent this is unanswerable and attempts by Duhaime et $a l^{3}$ using infant models and accelerometers tended to suggest that the deceleration experienced during impact injury was much greater than that from shaking. The association between ocular haemorrhage and intracranial haemorrhage has long been well known. As with intracranial haemorrhage, the degree of force required to cause the various ocular haemorrhages observed in NAI have been difficult to assess.

An important paper in the current issue of the $B \mathcal{B O}$ (Green et al, p 282) has made a substantial contribution towards answering this question. The authors have examined the eyes taken from 23 children dying of unequivocal NAI and, in addition, performed an extremely detailed forensic necropsy on each. Sixteen of 23 children died as the result of head injury. Of the remaining seven the injuries were to other parts of the body. All but one of the 16 who died of head injury had subdural haemorrhages which were solitary in eight of the cases but the remaining seven had more extensive intracranial trauma. The authors have constructed both an eye trauma score and an intracranial trauma score based on the presence of the number of different injuries found in any single individual. It has been possible, using the ocular trauma score, to make some kind of prediction as to the severity of the intracranial injuries likely to be found and of the degree of violence sustained. Eye injuries were found in $81 \%$ of those dying of head injuries but in only $17 \%$ of those where death was related to injuries elsewhere in the body. Ten of 16 patients with subdural haematomas had retinal detachment and these patients had a significantly greater number of other intraocular injuries than the six patients who had no retinal detachment.
The authors suggest that the same accelerationdeceleration forces that produce subdural haemorrhage also apply to the vitreous, producing subhyaloid and intraretinal haemorrhage and, in may instances, retinal detachment. They observed that these lesions occur at the periphery of the retina where the vitreous is strongly attached and emphasise the need for indirect ophthalmoscopy whenever a young child is suspected of having an intracranial injury, particularly if this is unexplained. Most usefully, they conclude that, with the least severe degree of head trauma, subdural haemorrhage alone may present, but with increasing force subhyaloid and intraretinal haemorrhages, with or without perineural optic nerve sheath haemorrhages, may occur. Greater degrees of violence will frequently produce retinal detachment. The most severe traumas - that is, choroid and vitreous haemorrhages, are likely to be associated with subarachnoid and intracerebral haemorrhages in addition to subdural haematoma.

From the paediatrician's point of view the authors point out that any intraocular injury may indicate the presence of a subdural haemorrhage and that, furthermore, if not already present, it may develop later. With regard to the controversy over whiplash injuries versus impact injuries, it would have been interesting to know how many of the dead children in this study had sustained skull fractures.

One of the authors' cases was found to have cerebral oedema as the only intracranial finding following shaking. This child had retinal detachment, indicating considerable force had been applied. The authors were unable to judge the presence of cerebral oedema in their other cases but this has been a prominent feature in at least one other study by Munger et $a l,{ }^{4}$ where $83 \%$ were found to have cerebral oedema. This is a not infrequent finding in shaking injuries and may be attributed to a primary hypoxic event, such as aspiration of stomach contents, by a defence counsel.

This is an important paper for any paediatrician or ophthalmologist who has to give evidence in court in relation to child abuse. It will draw attention to the need for enlisting the early help of an ophthalmologist for any child suspected of sustaining injury to the brain. Similarly, it will remind forensic pathologists to remove the eyes from those dying of intracranial trauma for further examination where criminal proceedings are contemplated and the degree of force used may become an issue.

The Royal Hospitals NHS Trust,

G J A I SNODGRASS

Garden House Children's Unit,

The Royal London Hospital,

Whitechapel, London E1 1BB

1 Caffey J. The whiplash infant syndrome: manual shaking infants, its potential residual effects of permanent brain damage and mental retardation. $A m \mathcal{J}$ residual effects of permanent

2 Hadley MN, Sonntag VKH, Rekate HL, Murphy A. The infant whiplashshake injury syndrome: a clinical and pathological study. Neurosurgery 1989; 24: 536-40.

3 Duhaime A-C, Gennarelli TA, Thibault LE, Bruce DA, Margulies SS, Wiser $R$. The shaken baby syndrome, a clinical pathological and biochemical study. 7 Neurosurg 1987; 66: 409-15.

4 Munger CE, Peiffer RL, Bouldin TW, Kylstra JA, Thompson RL. Ocular and associated neuropathologic observations in suspected whiplash shaken infant syndrome. A retrospective study of 12 cases. Am $\mathcal{f}$ Forensic Med Pathol 1993; 14: 193-200. 